\title{
In Vivo Inhibition of Multivitamin on the Formation of Hemoglobin Adduct in 4-Aminobiphenyl-Treated Rat
}

\author{
T. Qin, ${ }^{1}$ X. B. Xu, ${ }^{1}$ L. X. Zhao, ${ }^{1}$ Z. L. Jin ${ }^{2}$ \\ 1 Research Center for Eco-Environmental Sciences, Chinese Academy of Sciences, \\ Post Office Box 2871, Beijing 100085, People's Republic of China \\ 2 National Natural Science Foundation of China, Post Office Box 8610, Beijing \\ 100085, People's Republic of China
}

\section{Received: 26 January 2001/Accepted: 27 June 2001}

The relationships between the amounts of hemoglobin adducts formed in blood and the doses using different methods of administration with 4-aminobiphenyl (4-ABP) to rat were studied. Hemoglobin adducts in vivo in rat varied with the duration after exposure. With a dose of $1 \mu \mathrm{g} / \mathrm{kg}$ of 4-aminobiphenyl, the highest formation of hemoglobin adduct with 4-ABP in rat blood appeared within 10-15 day. The inhibition of multivitamin for the hemoglobin adduction with 4-ABP can be observed in rat in vivo. The variations of hemoglobin adduct with 4-ABP were $20-40 \%$ lower compared with the samples in the control groups in different durations when the administrations of 4-ABP and multivitamin were controlled at certain level. The impacts on the amounts of hemoglobin adduct with 4 -aminobiphenyl (4-ABP-Hb) were investigated by changing the way of administration and doses of 4-ABP to the test animals.

One of typical carcinogens among numerous poly-aromatic amines hydrocarbon was 4-aminobiphenyl that was extensively measured in blood samples collected from smokers who used various sorts of tobacco and cigarette (Bryant, MS 1988). It is an irrefutable fact that cigarette smokes harm the health of people who exposes to the smoke actively or passively (Maclure M 1990). The attack of diseases happened in heart or brain and the incidence of cancers occurred at lung, bladder or breast was evidently higher in the smokers than nonsmokers. The damage to a large number of people being suffered the exposure to environmental contamination should not be ignored. Evidence from the studies on the toxic and harmful chemicals in environment suggested that it was always accompanied to investigate carcinogens with animal test by long testing period and high cost. Therefore it is significant to assess by dealing with a simpler and short-term animal test as bio-marker to monitor the injure for health. Recent studies have defined that the formations of hemoglobin adduct with 4-ABP in maternal and fetal blood samples of smokers were obviously of higher values than in those of nonsmokers (Vineis et al. 1996). 
In this study we investigated the binding of hemoglobin to 4-ABP being as a xeno-biological compound in blood samples and the inhibition of multivitamin on the formation of hemoglobin adduct with 4-ABP in vivo in rat. The purpose was attempted to seek an available bio-marker to monitor the formation values and to observe the prevention role of multivitamin from potent tobacco-related carcinogen within animal experiment. Our results demonstrated that the concentrations of 4-ABP hemoglobin adducts in vivo in rat were related with the time period after the administration. In addition, the formations of 4-ABP hemoglobin adducts were impaired by the detoxifying effect of multivitamin with analyzing the blood samples of rats compared with controlled samples.

\section{MATERIALS AND METHODS}

Heptafluorobutyric anhydride (HFBA) was purchased from Fluka Chem. All solvents used such as n-hexane and toluene etc. were distilled and kept in glassware. N-hexane was distilled twice by a high efficiency facility and stored in clean glass bottles. All water used for preparation of aqueous solution such as sodium hydroxide and saline, and used in the dialysis procedure must be re-distilled from a dilute alkaline permanganate solution. Trimethylamine was from Shanghai Chemicals Factory (China). Anhydrous sodium sulfate came from Beijing Xinghua Chemical Factory (Beijing China) and was pretreated at a temperature above $550{ }^{\circ} \mathrm{C}$ before using. Heparinate used as anticoagulant and dialyzer were purchased from Sino-American Biotechnology Company (Beijing, China). Multivitamin formula with minerals was purchased from Gold Theragran Comp. (Shanghai, China). The dose of administration was dependent on the amount of $\beta$-carotene if the other vitamins were not changed. 4-Aminobiphenyl as standard and hemoglobin standard and quantitative assay reagents (Sigma Drabkin's Kit No. 525-A) were purchased respectively from Aldrich and Sigma Chemical Comp. (Beijing, China).

Analytical method of hemoglobin-4-aminobiphenyl adduct had been described (Steven et al, 1996). The parent 4-ABP hydrolyzed from 4-ABP hemoglobin adduct was determined by using Varian 3740 gas chromatograph equipped with electron capture detector. The GC column used was SE-54 capillary column ( $40 \mathrm{~m}$ $\times 0.25 \mathrm{~mm}$ internal diameter, $0.55 \mu \mathrm{m}$ film thickness). The column temperature program employed was starting temperature $50{ }^{\circ} \mathrm{C}$ for $2 \mathrm{~min}$., followed by $4{ }^{\circ} \mathrm{C}$ $\mathrm{min}^{-1}$ ramp to $280{ }^{\circ} \mathrm{C}$ final temperature, held for $20 \mathrm{~min}$. Nitrogen was used as carrier. Quantification of the parent 4-ABP hydrolyzed from hemoglobin adduct was determined by comparing the relative peak area of GC-ECD chromatogram of HFBA derivative of 4-ABP with the calibration curve of 4-ABP standard. The 
limit of detection for 4-ABP-Hb adduct was $0.28 \mathrm{ng}^{-1}(\mathrm{Hb})$. The spectrophotometer (made in Shanghai Analytical Instrument Company, China) was used to determine the total amount of hemoglobin in blood samples. The hemoglobin separated from blood sample was performed by high speed centrifugation (J2-HS Backman) at 10,000 $\mathrm{g}$ for $20 \mathrm{~min}$.

Adult female SD rat of weight $200 \pm 25 \mathrm{~g}$ were selected from the experimental animals feeding at Beijing Medical University. They were housed separately in cages and maintained under standard laboratory conditions (room temperature, 25 $\pm 5^{\circ} \mathrm{C}$ and relative humidity, $50-60 \%$ ). They were randomly divided into groups of three rats and they were administered by the same way, fed on commercial food and offered tap water ad libitum. Each point in the results was the average of three rats in each group. 4-ABP was dissolved in corn oil. A maximum of $0.3 \mathrm{ml}$ of $0.59 \mathrm{mmol} \mathrm{kg}$-1 was need in the peritoneal injection while a maximum of 0.4 was administered the stomach instillation. Multivitamin was fed by gavage to rats between 8:30 to 9:30 am each day except for the control group. All rats had free access to feed and water throughout. The blood sample each about 5-8 $\mathrm{ml}$ was obtained by heart puncture with heparinized syringe after the animals were anesthetized with ether. The blood samples were processed using the method of Bryant M.S. et al 1987 and the method described by Drabkin's assay (Qin, T. et al, 1997).

\section{RESULTS AND DISCUSSION}

In the present study the analysis of some hemoglobin adducts was recommended as a dosimeter in biological monitoring of humans in order to control exposure. It has been possible to study the association between tobacco smoke exposure and carcinogen induced DNA damage due to the advances in quantitative analysis of covalent adducts. Recently the formation of hemoglobin adducts with various environmental compounds was proposed as a potential bio-marker of exposure to carcinogenic compounds (Steven R.M. et al, 1996, Bartsch et al, 1990), There were several reports concerning numerous aromatic amines detected in tobacco smoke. Some results demonstrated that these amines were potent human bladder carcinogens especially including 4-aminobiphenyl and 2-naphthylamine. Only few experiment about exposure studies on such carcinogens were reported in literature meanwhile concerning the detoxification by antioxidant or other xeno-biological materials. Therefore it is important to focus on the inhibition of multivitamin on the formation of hemoglobin adduct with 4-aminobiphenyl in animal test. Since some of aromatic amines were potent human bladder carcinogens, it was a reasonable hypothesis that the increased formation of 
hemoglobin adduct with carcinogens was a primary risk factor in the incidence of bladder cancer (Skipper et al, 1990).

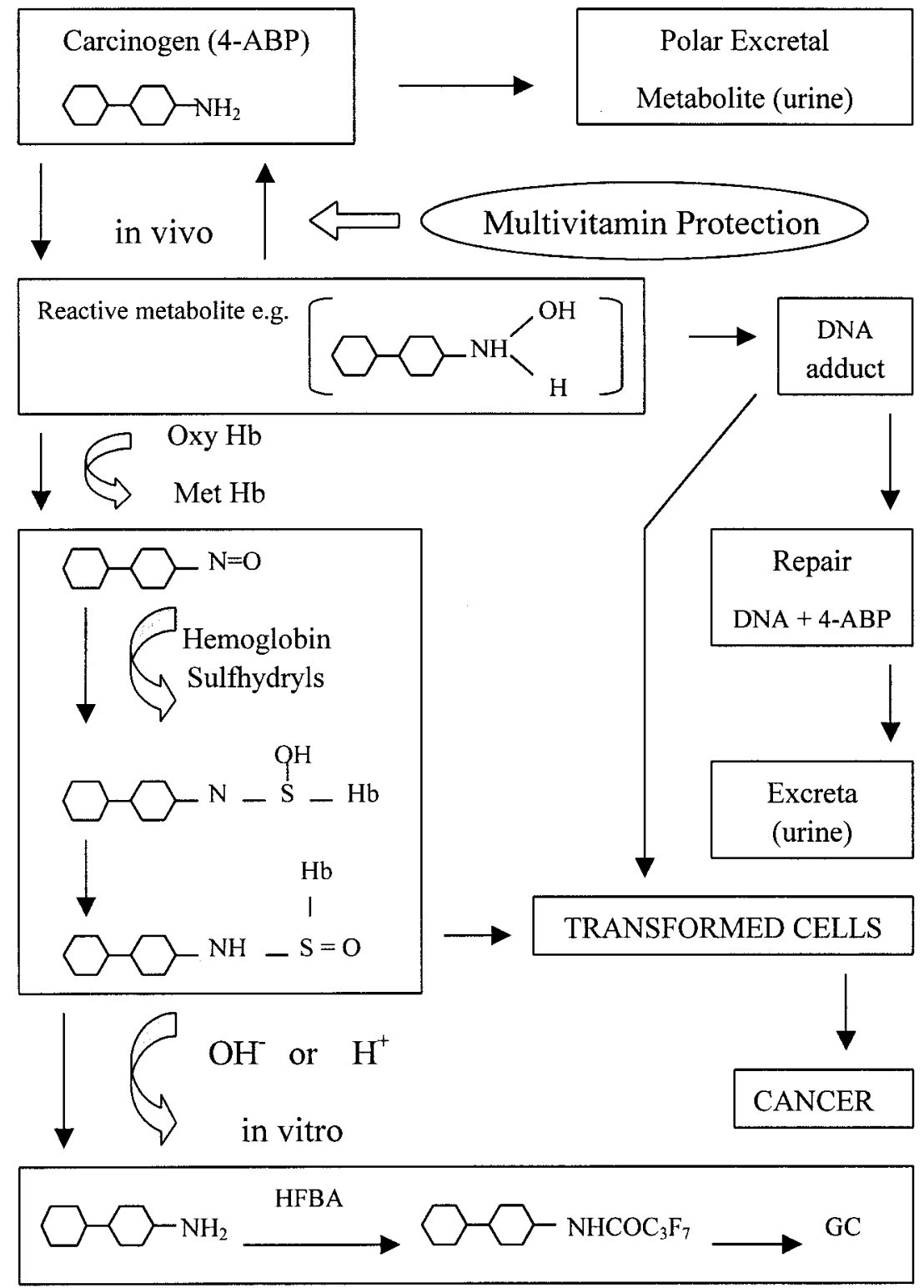

Figure 1. Metabolism of carcinogen 4-aminobiphenyl (4-ABP) leading to hemoglobin and DNA adducts and inhibition of multivitamin in 4-ABP-treated rat in vivo and processes for assay of hemoglobin adduct with 4-aminobiphenyl $(4-\mathrm{ABP}-\mathrm{Hb})$ in vitro 


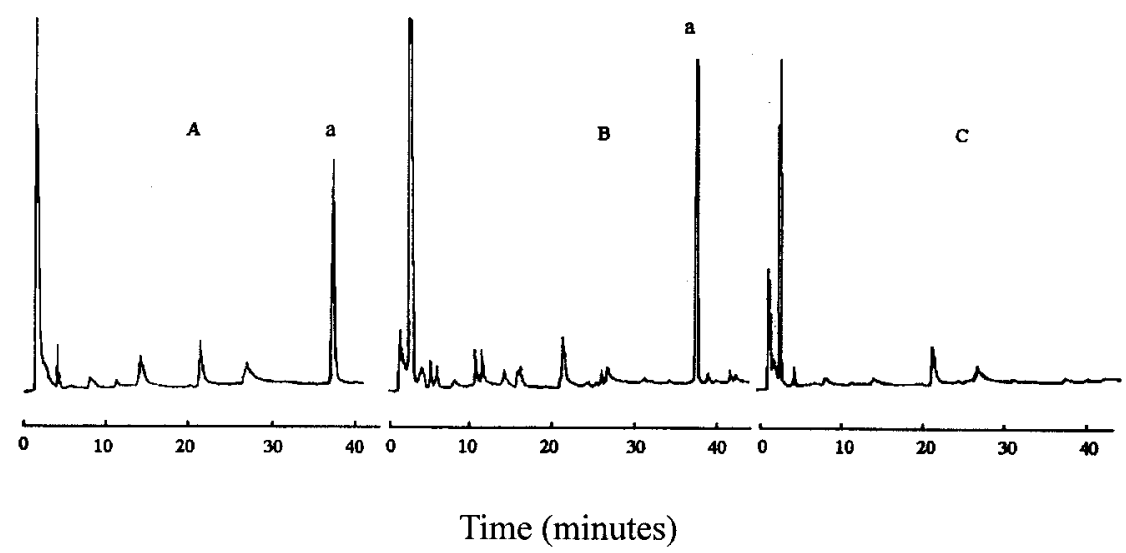

Figure 2. GC-ECD chromatograms of heptafluorobutyric anhydride (HFBA) derivative of 4-aminobiphenyl (4-ABP) and of the hydrolysate from $\mathrm{Hb}$ adduct (A: 4-ABP-HFBA standard, B: adduct to $\mathrm{Hb}$ in rat blood, $\mathrm{C}$ : blank sample of rat blood, a: derivative)

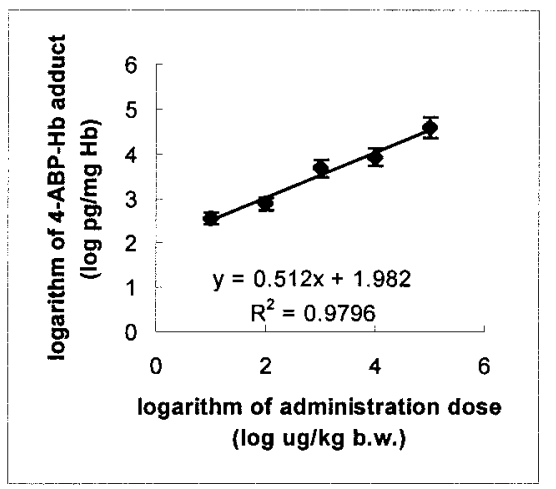

Figure 3. Formation curve of hemoglobin adducts with 4-aminobiphenyl (4-ABP-Hb) in vivo in rat blood by administration with peritoneal injection

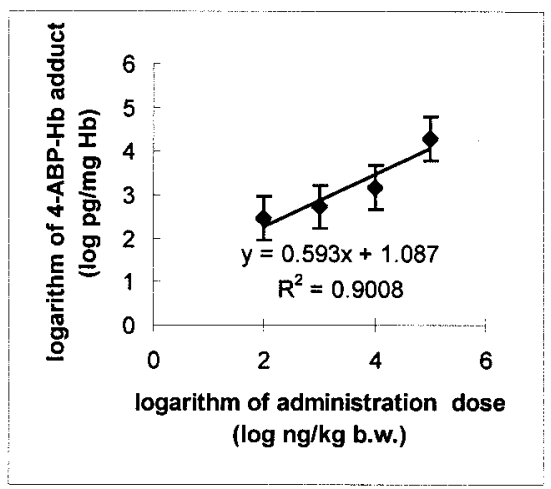

Figure 4. Formation curve of hemoglobin adducts with 4-aminobiphenyl (4-ABP-Hb) in vivo in rat blood by administration with stomach instillation 


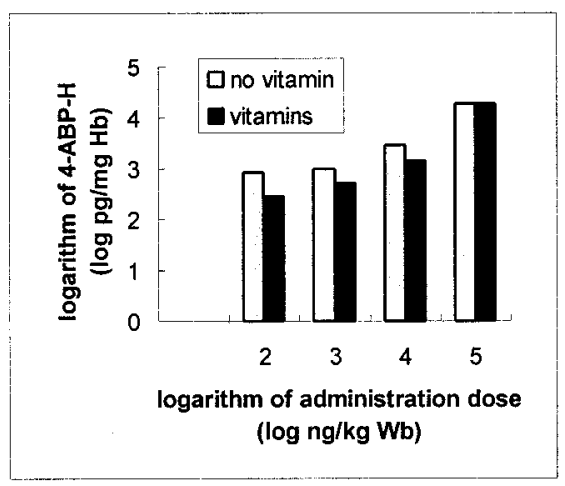

Figure 5. Effect of multivitamin of equal dose and various doses of 4-aminobiphenyl (4-ABP) treated to rat by stomach-instillation on the formation of $4-\mathrm{ABP}-\mathrm{Hb}$ adducts

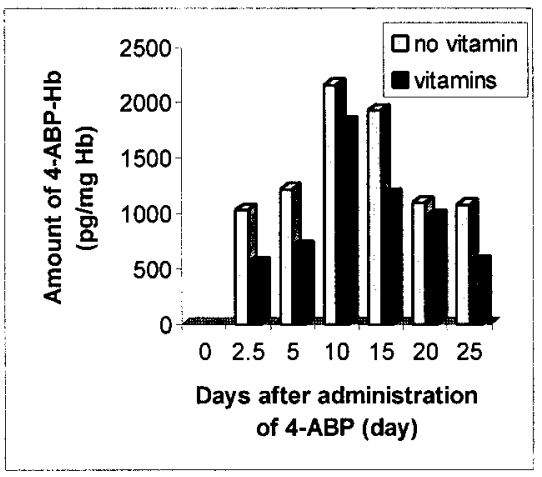

Figure 6. Effect of multivitamin addition on the formation of $4-\mathrm{ABP}-\mathrm{Hb}$ adducts within 25 days after same dose of $1 \mu \mathrm{g} \mathrm{Kg}^{-1}$ (b.w.) administration of 4-aminobiphenyl (4-ABP) treated to rat by stomach-instillation

The hemoglobin adducts with 4-ABP in vivo formed in the metabolism and the multivitamin inhibition role for the formation of hemoglobin adduct are shown in Figure 1. The results of analysis by GC-ECD in Figure 2 were obtained from the 4-ABP-treated rat blood samples pretreated with the methods described above.

In vivo test the animals were randomly divided into six groups in which each group included three rats. One group was used as control group that was injected corn oil only, 4-ABP was administrated by peritoneal injection to other five groups, the exposure levels were $10,100,1000,10000,100000 \mu \mathrm{g} \mathrm{kg}^{-1}$ (b.w.) respectively. Since the administration, 65 hours later the blood samples were taken by heart puncture method to assay the hemoglobin adduct values. The relationship between the logarithm of the values of $4-\mathrm{ABP}-\mathrm{Hb}$ adduct in blood and the logarithm of the administration levels is shown in Figure 3 and the coefficient is $r^{2}=0.9796$.

In another test, we used the same way to deal with the rats divided into groups, but the method of administration was different. Four groups of rats were administrated by stomach-instillation, except for one group as contrast one feeding corn oil without any 4-ABP. The exposure levels were $0.1,1.0,10,100$ $\mu \mathrm{g} \mathrm{Kg}{ }^{-1}$ (b.w.) respectively. After 62 hours, the blood samples collected by 
heart puncture were determined and the results of hemoglobin adduct values relevant to the amounts of administration are shown in Figure 4. Comparing the two different administration methods, the values of hemoglobin adduct with 4-ABP were obviously higher by stomach instillation than by peritoneal injection with the same level of exposure.

For the purpose of studying the effect of inhibition of multivitamins on the formation of hemoglobin adduct with 4-aminobiphenyl in rat, the animals were divided into two series of groups, except for the group of control, the two series of groups of rats were treated with 4-ABP by stomach-instillation, and the doses of 4-ABP were $0.1,1.0,10,100 \mu \mathrm{g} \mathrm{K}^{-1}$ (b.w.) respectively. The way of administration and the amount of exposure were the same for the two series. The rats of one series were administrated the same amount of multivitamins which contained $\beta$-carotene at the same level of $7.8 \times 10^{3} \mathrm{I} . \mathrm{U} . \mathrm{Kg}^{-1}$ (b.w.), this dose was administrated every day until the day of blood sample collecting, and they were used from the day before exposure to 4-ABP. But the rats in the other series were never administrated with multivitamin at all. Figure 5 shows that the amounts of hemoglobin adduct with 4-ABP in the blood of rats without any use of multivitamins were always higher than the other series of rats fed with multivitamins every day.

The same way of dividing rat groups and administration of 4-ABP and multivitamins were used in another experiment. The administration of 4-ABP to rat was $1 \mu \mathrm{g} \mathrm{Kg}{ }^{-1}$ (b.w.) only one time. Every day the rats were fed with multivitamins containing $8.3 \times 10^{3} \mathrm{I}$.U. $\mathrm{Kg}^{-1}$ (b.w.) $\beta$-carotene from two days before the administration of 4-ABP until killed. The blood samples collected at different time period were assayed. Figure 6 shows the values of hemoglobin adduct with 4-ABP in blood samples of rats which all had been exposed to 4-ABP but some treated with multivitamins and others did not. The highest levels of hemoglobin adduct with 4-ABP in blood of rats were observed about 10 or 15 days after exposure. The 4-ABP-Hb adduct value decreased by feeding multivitamins or the inhibition of multivitamins in rat in vivo were $20 \%-40 \%$ as compared with the control samples for the same exposure and same time period. Using the assay of the values of hemoglobin adduct in blood of rats we can study the roles of the anti-carcinogens or the inhibitor in vivo in rat. It may give an evidence that the formation level of hemoglobin adduct in vivo can be used as bio-markers to monitor exposure levels or as a method to find a chemical protection trial or a medication.

Acknowledgments. This study was partially supported by the grant from the National Natural Science Foundation of China (NSFC 29837180) and the 
Chinese Academy of Sciences (KJ 952-51-520 CAS, RCEES 9906).

\section{REFERENCES}

Bartsch H, Caporaso N, Coda M, Kadlubar F, Malaveille C, Skipper P, Talaska G, Tannenbaum SR, Vineis P (1990) Carcinogen hemoglobin adducts, urinary mutagenicity, and metabolitic phenotype in active and passive cigarette smokers. J Natl Cancer Inst 82: 1826-1830

Bryant MS, Skipper PL, Tannenbaum SR Maclure M (1987) Hemoglobin adducts of 4-aminobiphenyl in smokers and nonsmokers. Cancer Res 47: 602-608

Bryant MS, Vineis P, Skipper PL, Tannenbaum SR (1988) Hemoglobin adducts of armatic amines: associations with smoking status and type of tobacco. Proc Natl Acad Sci USA 85: 9788-9791

Maclure M, Bryant MS, Skipper PL (1990) Decline of the hemoglobin adducts of 4-aminobiphenyl during withdrawal from smoking. Cancer Res 50: 181-184

Qin T, Zhao LX, Xu XB (1997) In vivo formation of 4-aminobiphenyl adduct with hemoglobin in rat (Chinese). Acta Scientiae Circumstantiae 19: 667-671

Skipper PL, Tannenbaum SR (1990) Protein adducts in the molecular dosimetry of chemical carcinogens. Carcinogenesis 11: 507-518

Steven R Myers, Joseph A Spinnato, Maria T Pinorini-Goldly, Curtis Cook, Brent Boles, George C Bodgers (1996) Characterization of 4-aminobiphenyl-hemoglobin adducts in maternal and fetal blood samples. J Toxicol Environ Health 47: 553-566

Vineis P, Talaska G, Malaveille C, Bartsch H, Martone T, Sithisarankul P, Strickland P (1996) DNA adducts in urothelial cells: Relationship with biomarkers of exposure to arylamines and polycyclic aromatic hydrocarbons from tobacco smoke. Int J Cancer 65: 314-316 